\title{
Aprovechamiento del grano de café verde (Coffea arabica) en la elaboración de una bebida refrescante
}

\section{Use of green coffee grain (Coffea arabica) in the preparation of a refreshing drink}

\author{
MSc Johanna Isabel Llerena Moreno ${ }^{1}$ \\ imjohisallm@gmail.com \\ Ing. Carlos Santiago Solís Chamorro ${ }^{2}$ \\ sansolis05@gmail.com \\ MSc. Hitler Abdón Guerrero Hidalgo ${ }^{2}$ \\ Hitgh1447@gmail.com
}

Recibido: 1/04/2018, Aceptado: 1/06/2018

\begin{abstract}
RESUMEN
La presente investigación tuvo como objetivo el desarrollo de una alternativa tecnológica que permita el aprovechamiento de las propiedades funcionales del café verde en la elaboración de una bebida refrescante. Mediante un enfoque cualitativo y alcance correlacional. La muestra tomada fue de la provincia de Loja, se trabajó con $18 \mathrm{~kg}$ de café verde de la variedad arábiga. Los factores de estudio considerados fueron la concentración de café, tiempo de infusión y la concentración de ácido cítrico. La selección del mejor tratamiento es mediante análisis estadístico y un panel de catadores semi entrenados (análisis sensorial). Los resultados transcendentales demuestran que permitió obtener una bebida dentro de las especificaciones de la norma INEN 2 411:2008 (318 mg de cafeína/L) y una menor variación en el índice de oscurecimiento $(22,41)$; además presentó las mejores características sensoriales (color, olor, sabor y aceptabilidad) según el panel de catadores. La estabilidad de la bebida en condiciones aceleradas $\left(17^{\circ} \mathrm{C}\right.$ y $\left.65 \%\right)$ en función de sus parámetros microbiológicos y químicos (turbidez y funcional) determinando que está puede alcanzar una vida de anaquel de 18 días.
\end{abstract}

Palabras clave: arábica, café verde, características sensoriales, propiedades funcionales

\footnotetext{
1 Universidad Técnica de Ambato, Ecuador

2 Escuela Superior Politécnica de Chimborazo, Ecuador
} 


\begin{abstract}
The objective of this research was to develop a technological alternative that allows the use of the functional properties of green coffee in the production of a refreshing drink. Through a qualitative approach and correlational scope. The sample taken was from the province of Loja, we worked with $18 \mathrm{~kg}$ of green coffee of the Arabian variety. The study factors considered were the concentration of coffee, time of infusion and the concentration of citric acid. The selection of the best treatment is through statistical analysis and a panel of semi-trained tasters (sensory analysis). The transcendental results show that it allowed obtaining a drink within the specifications of the INEN 2 411: 2008 standard (318 mg of caffeine / L) and a smaller variation in the darkening index $(22,41)$; also presented the best sensory characteristics (color, smell, taste and acceptability) according to the panel of tasters. The stability of the drink under accelerated conditions ( $17^{\circ} \mathrm{C}$ and $\left.65 \%\right)$ depending on its microbiological and chemical parameters (turbidity and functional) determining that it can reach a shelf life of 18 days.
\end{abstract}

Keywords: arabica, green coffee, functional properties, sensory characteristics

\title{
Introducción
}

En los últimos años se vienen desarrollando en el mundo propuestas de productos alimenticios con características diferentes a las fisicoquímicas y sensoriales, es decir, alimentos fisiológicamente funcionales; por tal motivo, se ha venido investigando el café, su composición, propiedades y su relación con la salud. Actualmente se conoce que este producto agroalimentario posee una serie de bioactividades, como actividad antioxidante, anticarcinogénica y antimutagénica. Los granos de café verde contienen antioxidantes como ácidos fenólicos, polifenoles y alcaloides; especialmente los ácidos fenólicos elágico, cafeico y clorogénico; el contenido de estos componentes varía entre especies y lugar de origen y le dan al café la calidad de alimento funcional y nutracéutico (Naranjo, Vélez y Rojano, 2011).

El café es fuente de múltiples historias, leyendas, tradiciones y aceptado como una bebida universal; en la actualidad se denomina así a la semilla de las bayas que produce el cafeto (Coffea spp.); inicialmente estas son de color verde, pero adquieren un color rojo carmesí al madurar (Almazara, 2012). Deriva su nombre del árabe "kahawh", "Kawa" o "gawah" traducido al español significa "amargo" (Guilcapi, 2009).

Existe un consenso casi unánime, acerca del origen del café; se habla de un cultivo silvestre conocido como Arábica en el altiplano de Abisinia (actual Etiopia), y se atribuye dicho descubrimiento a Kaldi (850 D.C.), un pastor de la localidad quien observó que sus ovejas estaban especialmente eufóricas y saltaban llenas de energía después de haber comido las hojas y frutos de cierto arbusto. Este efecto suscitó la curiosidad de los monjes locales, estos colocaron las cerezas al fuego y al tostarse produjeron un exquisito aroma. Mediante la comunicación de estos efectos a conventos vecinos, el café se difundió con rapidez por todo el mundo (Almazara, 2012). 


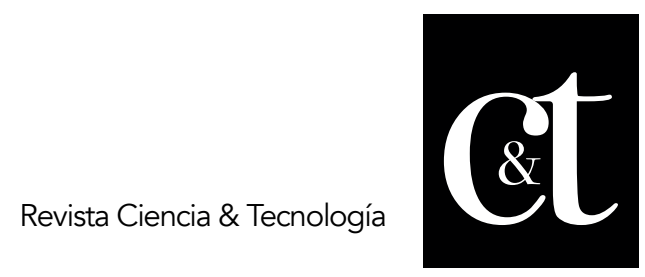

No. 19, 31 de julio de 2018

ISSN impreso: 1390 - 6321

Con el tiempo se convirtió en una de las bebidas más populares y consumidas en todo el mundo, por lo que cada región la adopto de maneras diferentes creando recetas propias.

Actualmente, el café es la bebida psicoactiva más ingerida, que más allá, de un efecto estimulante es capaz de promover la salud y prevenir enfermedades. Según diversos estudios una ingesta moderada tiene efecto protector para la prevención y/o tratamiento de diversas patologías como: el Ictus, Alzheimer y Parkinson; además, combate problemas neurológicos, cardiacos, oncológicos, hepáticos, endocrinos, renales, óseos y reduce el riesgo de enfermedades vasculares (Cirera, 2009).

El café es uno de los productos de mayor facturación en el mundo después del petróleo y el acero a nivel mundial (Cortijo, 2008).

\section{Desarrollo}

Para Arguedas (2013), el aprovechamiento del grano de café o también llamado el grano de oro, permite optimizar todos los residuos que se obtienen, con el propósito de disminuir desechos, además de conservar todas características funcionales como sensoriales que posee el mismo en una bebida refrescante.

\section{Marco Referencial}

Café

Según Mora (2008) el café pertenece al género Coffea con aproximadamente 100 especies. No obstante, únicamente tres de éstas se mencionan son cultivadas comercialmente, destacándose las dos primeras según el orden siguiente: Coffea arabica L., C. Canephora Pierre exFroehner y C.liberica Bull exHiern.

\section{Catación y características organolépticas}

La catación es una prueba que consiste en analizar de formas sistémica y cuantificada las particularidades organolépticas y sensoriales del café. Esta evaluación la realiza un catador, que es una persona con amplios conocimientos, tiene experiencia y habilidades para percibir los atributos y defectos del café. Sin omitir que los aspectos evaluados son subjetivos ya que se fundamentan en la opinión y preferencias del catador (Alfaro, 2015). Vida útil de los productos de café El seguimiento de la vida útil de los productos de café se podría decidir por determinar únicamente el contenido de fenoles totales y su actividad antioxidante por el método de ABTS que es un radical artificial que no mimetiza bien la situación in vivo. Sin embargo, la mayoría de los artículos que caracterizan la actividad antioxidante en las bebidas de café utilizan varias metodologías (Londoño, Naranjo y Quintero, 2013).

\section{Cafeína}

La cafeína, fue aislada del grano de café en 1820 (Cano, Tarín y Cano, 2013). También denominada guaranina o teína, es un alcaloide del grupo de las xantinas (1,3,7- trimetilxantina). Actúa como una droga psicoactiva y estimulante debido a su acción antagonista no selectiva de los receptores de adenosina. Es por ello que a la cafeína se la puede considerar la sustancia estimulante de mayor consumo y 
mejor aceptación social a nivel mundial.

Determinación de propiedades físicas y mecánicas

Se determinaron propiedades físicas o mecánicas de las diferentes estructuras del árbol de café, que se puedan tomar como base para el desarrollo exitoso de tecnologías que realicen más ágilmente el desprendimiento de los frutos maduros, la dificultad se debe principalmente a factores tales como una relación fuerza de desprendimiento sobre peso de los frutos muy alta, pedúnculo con muy buenas características estructurales, frutos muy apretados en los glomérulos, árboles con corteza muy débil, tronco de los árboles y sus ramas muy flexibles (Tascón y Saenz, 2011).

\section{Metodología}

Recopilación de datos, descripción y evaluación de la muestra

La concentración de extracto de café verde, tiempo de infusión y ácido cítrico influye en las características funcionales y sensoriales de la bebida.

Mediante un enfoque cualitativo y alcance correlacional. La muestra tomada fue de la provincia de Loja, se trabajó con $18 \mathrm{~kg}$ de café verde de la variedad arábiga. Los factores de estudio considerados fueron la concentración de café, tiempo de infusión y la concentración de ácido cítrico. La selección del mejor tratamiento es mediante análisis estadístico y un panel de catadores semientrenados (análisis sensorial).

\section{Resultados}

El análisis estadístico permitió identificar el mejor tratamiento a nivel experimental y sensométrico. Dentro de los parámetros fisicoquímicos estudiados se determinó que existe una relación directa entre la concentración de café verde y el tiempo de infusión (min) sobre la concentración de cafeína, estableciéndose que un tiempo prolongado de infusión incrementa la concentración del alcaloide, un efecto similar se observa en cuanto a la concentración de café verde puesto que una mayor cantidad de café incrementa el contenido de cafeína en las bebidas desarrolladas.

Los cambios de tonalidad de la bebida (índice de oscurecimiento) presentaron una relación directa entre la concentración de café verde y la concentración de ácido cítrico; presentando una relación directa sobre el incremento del índice de oscurecimiento. En concentraciones inferiores a 1000 ppm se observa una mayor variación de tono, determinándose que en los tratamientos con mayor contenido de café este proceso es más marcado que en muestras con un menor contenido de café crudo.

A continuación se presentan los resultados obtenidos mediante el análisis estadístico y las pruebas sensoriales se estableció que la concentración de extracto de café verde, el tiempo de infusión y ácido cítrico influyen en las características funcionales y sensoriales de la bebida, $a$ un nivel de significancia de $95 \%(a=0,05 ; p>0,05)$, los Análisis de varianza para el contenido de cafeína, el índice de oscurecimiento y las características sensoriales respectivamente, aceptándose la hipótesis alternativa que indica que el efecto combinado de los factores de estudio influyen en el desarrollo de la bebida y que no existe un efecto individual de estos sobre la 


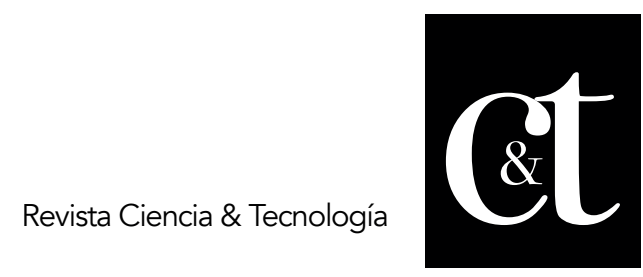

No. 19, 31 de julio de 2018

ISSN impreso: 1390 - 6321

formulación desarrollada y sus propiedades organolépticas.

Tabla 1. Análisis de varianza del índice de obscurecimiento en bebidas refrescantes a base de café

\begin{tabular}{|l|r|r|r|r|r|}
\hline \hline Factor & \multicolumn{1}{l|}{ SS } & Df & \multicolumn{1}{l|}{ MS } & F & P \\
\hline (1) Conc. Café verde (L) & 81,664 & 1 & 81,6639 & 0,304713 & 0,604707 \\
\hline Conc. Café verde (Q) & 0,284 & 1 & 0,2841 & 0,00106 & 0,975287 \\
\hline (2) Tiempo de infusión (L) & 228,679 & 1 & 228,6788 & 0,853271 & 0,398017 \\
\hline Tiempo de infusión (Q) & 23,536 & 1 & 23,5364 & 0,087822 & 0,77888 \\
\hline (3) Ác. Cítrico (Q) & 2,714 & 1 & 2,7142 & 0,010128 & 0,92375 \\
\hline Conc. Ác. Cítrico (Q) & 38,669 & 1 & 38,6695 & 0,719655 & 0,144288 \\
\hline 1L by 2L & 15,905 & 1 & 15,9048 & 0,059346 & 0,817213 \\
\hline 1L by 3L & 341,65 & 1 & 341,6498 & 1,2748 & 0,310108 \\
\hline 2L by 3L & 9,374 & 1 & 9,3744 & 0,034979 & 0,858992 \\
\hline Error & 1340,013 & 5 & 268,0027 & & \\
\hline Total SS & 2090,997 & 14 & & & \\
\hline
\end{tabular}

Fuente: elaboración propia

Se determinó que no existe un efecto significativo individual de la concentración de café verde (\%), el tiempo de infusión (min) y la concentración de ácido cítrico en el desarrollo de la bebida puesto que los valores $p$ son superiores a 0,05 para todos los factores y las interacciones; de tal modo que tanto los términos lineales y los cuadráticos de los tres factores son importantes para el desarrollo de la bebida.

Tabla 2. Análisis de varianza para la Concentración de cafeína para bebidas refrescantes de café verde

\begin{tabular}{|l|r|r|r|r|r|}
\hline Factor & \multicolumn{1}{l|}{ SS } & Df & MS & F & P \\
\hline $\begin{array}{l}\text { (1) Conc. Café verde } \\
(\%)(L)\end{array}$ & 3252,3 & 1 & 3252,3 & 0,105434 & 0,758549 \\
\hline Conc. Café verde (\%)(Q) & 22835,6 & 1 & 22835,6 & 0,740299 & 0,428883 \\
\hline $\begin{array}{l}\text { (2) Tiempo de infusión } \\
(\%)(L)\end{array}$ & 103552,7 & 1 & 103552,7 & 3,357034 & 0,126406 \\
\hline Tiempo de infusión (\%)(L) & 20989,2 & 1 & 20989,2 & 0,680442 & 0,446988 \\
\hline (3) Ác. Cítrico (\%)(Q) & 11813,4 & 1 & 11813,4 & 0,382974 & 0,563118 \\
\hline Ác. Cítrico (\%)(Q) & 3135 & 1 & 3135 & 0,101632 & 0,762767 \\
\hline 1L by 2L & 7049,7 & 1 & 7049,7 & 0,228541 & 0,652777 \\
\hline 1 L by 3L & 163518,1 & 1 & 163518,1 & 5,301027 & 0,069564 \\
\hline 2L by 3L & 55,2 & 1 & 55,2 & 0,001791 & 0,967884 \\
\hline Error & 154232,5 & 5 & 30846,5 & & \\
\hline Total SS & 506065,8 & 14 & & & \\
\hline
\end{tabular}

Fuente: elaboración propia 
Con respecto a la concentración de cafeína, se estableció que no existe un efecto significativo individual de la concentración de café verde (\%), el tiempo de infusión (min) y la concentración de ácido cítrico en el desarrollo de la bebida puesto que los valores $p$ son superiores a 0,05 como se muestra en la tabla $n^{\circ} 2$, para todos los factores y las interacciones; de tal modo que tanto los términos lineales y los cuadráticos de los tres factores son importantes para el desarrollo de la bebida.

Tabla 3. Análisis de varianza de las características sensoriales obtenidas de la bebida refrescante a base de café verde

\begin{tabular}{|c|c|c|c|c|c|c|c|c|c|c|c|c|c|c|c|c|c|}
\hline & \multirow{2}{*}{ GL } & \multicolumn{3}{|c|}{ COLOR } & \multicolumn{3}{|c|}{ OLOR } & \multicolumn{3}{|c|}{ ASTRINGENCIA } & \multicolumn{3}{|c|}{ SABOR } & \multicolumn{3}{|c|}{ ACEPTABILIDAD } & \multirow{2}{*}{ FV } \\
\hline & & sc & $\mathrm{CM}$ & RV & sc & CM & RV & sc & CM & RV & sc & CM & RV & SC & CM & RV & \\
\hline Trat. & 14 & 20,84 & 1,489 & 0,23 & 16,8 & 1,2 & 0,325 & 11,11 & 0,794 & 0,98 & 16,16 & 1,154 & 0,761 & 20,76 & 1,483 & 0,4 & 1,87 \\
\hline Cat. & 34 & 28,19 & 0,829 & 0,413 & 26,13 & 0,769 & 0,508 & 29,52 & 0,868 & 0,896 & 30,8 & 0,906 & 0,969 & 30,63 & 0,901 & 0,659 & \\
\hline Resid. & 56 & 19,16 & 0,342 & 21,87 & 0,39 & 43,56 & 0,778 & 49,18 & 0,878 & 33,24 & 0,594 & & & & & & \\
\hline Total & 104 & 68,19 & & & 64,8 & & & 84,19 & & & 96,13 & & & 84.6 & & & \\
\hline
\end{tabular}

Fuente: elaboración propia

El análisis de varianza de las características sensoriales (color, olor, astringencia, sabor y aceptabilidad) para las 15 formulaciones desarrolladas, determinándose que estadísticamente no existe un efecto altamente significativo (Ft: $1,87<\mathrm{Fc}$ : 0,23; $0,413 ; 0,325 ; 0,508 ; 0,98 ; 0,896 ; 0,761 ; 0,969 ; 0,4$ y 0,659$)$ de la concentración del café $(\%)$, el tiempo de infusión (min) y la concentración de ácido cítrico (ppm) a un nivel de significancia del $5 \%(a=0,05)$, en la ponderación asignada a cada tratamiento; esto puede ser atribuido al efecto de la adición de una concentración estándar de stevia $(10 \mathrm{~g})$ y saborizante de manzana verde $(0,7 \mathrm{ml})$ en la bebida $(1$ L), lo que enmascara el efecto de las sustancias astringentes responsables del amargor del café verde y la acidez de los ácidos cítrico y ascórbico.

Tabla 4. Tiempo de vida útil de la bebida refrescante de café verde en función de la turbidez y el deterioro de polifenoles totales

\begin{tabular}{|c|c|c|c|c|c|c|}
\hline Deterioro & Pendiente & $\begin{array}{l}\text { Punto de } \\
\text { corte }\end{array}$ & $\begin{array}{l}\% \\
\text { Deterioro }\end{array}$ & Horas & Días & Meses \\
\hline Turbidez & 0,0124 & 2,7181 & 5,99 & 263,22 & 10,97 & 0,37 \\
\hline Funcional & $-0,0009$ & 7,1331 & 6,44 & 774,34 & 32,26 & 1,08 \\
\hline
\end{tabular}

Fuente: elaboración propia

Posteriormente se evaluó la estabilidad de la bebida considerando parámetros como turbidez y degradación de polifenoles totales; estableciéndose que a condiciones aceleradas $\left(17^{\circ} \mathrm{C}\right.$ y $65 \%$ de humedad relativa), esta presenta un incremento acelerado de la turbidez; esto puede ser atribuido a procesos de deterioro del edulcorante acalórico empleado.

La vida útil de la bebida se mantiene a través del tiempo observando una ligera disminución a partir de los 32 días de almacenamiento. 


\begin{abstract}
Discusión
Adicionalmente, en la concepción moderna de alimentos con declaraciones de propiedades nutricionales y de salud, es necesaria la estandarización de los productos en cuanto a su composición química, garantizando la estabilidad de los componentes bioactivos durante su vida útil, hasta llegar al consumidor final (Londoño, Naranjo y Quintero, 2013). La importancia de incluir nuevos parámetros no considerados hasta ahora en los estudios de vida útil de productos de café, especialmente aquellos relacionados con las declaraciones de contenido de antioxidantes (Blandón, Rodríguez y Dávila, 1998).
\end{abstract}

La calidad de un alimento está relacionada generalmente con su valor nutritivo y con el gusto obtenido durante la ingestión, así como con la ausencia de defectos en su presentación, sin que se perciba degradación de sus componentes o alteración en el sabor, la definición o ausencia de control de contaminantes químicos u orgánicos, para la comercialización y valoración del café, las características sensoriales son más importantes que el valor nutritivo.

\title{
Conclusiones
}

El trabajo investigativo proporciona una visión expectante para generar una alternativa tecnológica para el aprovechamiento de café verde (Coffea arabica) en la elaboración de una bebida refrescante, con propiedades funcionales por su aporte de cafeína natural y polifenoles propios del grano de café verde; además de la adición de un edulcorante acalórico; que lo convierte en una alternativa de consumo para segmentos de mercado exigentes con regímenes especiales de alimentación.

Además, el mejor tratamiento obtenido desarrollado contiene $4 \%$ de café verde, un tiempo de infusión de 10 minutos y 1000 ppm de ácido cítrico, permitiendo obtener una formulación dentro de los parámetros de calidad establecidos en la normativa nacional (Norma NTE INEN 1081:1984) en cuanto a la concentración de cafeína $318 \mathrm{mg} / \mathrm{L}$ y con un índice de oscurecimiento de 22,41 .

El tiempo de vida útil estimado del mejor tratamiento seleccionado es de 18 días, considerando una disminución de polifenoles y un incremento de turbidez. El porcentaje de deterioro por la turbidez es de 5,99 disminuyendo la vida de anaquel del producto hasta en 0,37 meses, los polifenoles se mantienen y tienen una ligera disminución a partir de los 18 días de almacenamiento

\section{Referencias bibliográficas}

Alfaro, V. (2015). Efectos de la altitud sobre las características físicas y organolépticas del café de la zona de los Santos (tesis de pregrado). Universidad de Costa Rica, San José, Costa Rica.

Almazara, E. (2012). De viandas y brebajes: café. México.

Arguedas, P. (2013). Definición del proceso de elaboración de una bebida fermentada a partir de pulpa del café (broza). Costa Rica, Cartago.

Blandón, G., Rodríguez, N., y Dávila, M. (1998). Caracterización microbiológica y física química de los subrproductos del beneficio del café en proceso de 


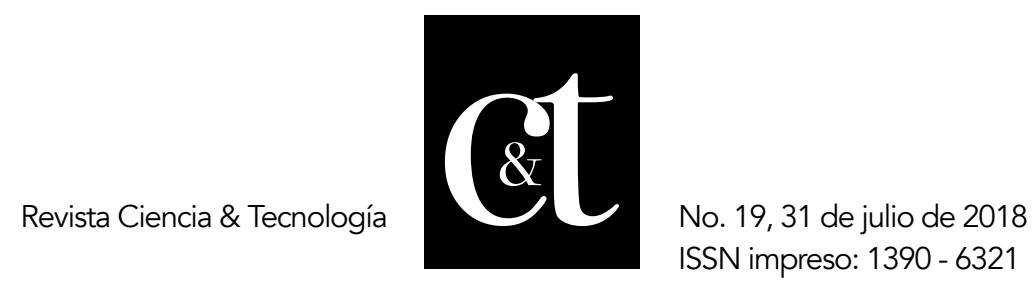

compostaje. Cenicafé, 49(3), 1-17.

Cano, A., Tarín, J., y Cano, A. (2013). The impact of coffee on health. Maturitas, 75(1), 7-21.

Guilcapi, E. (2009). Efecto de trichoderma harzianum y trichoderma viride, en la producción de plantas de café (coffea arábica) variedad caturra a nivel de vivero. (tesis de pregrado). Escuela Superior Politécnica de Chimborazo, Riobamba, Ecuador.

Londoño, J., Naranjo, M., y Quintero, M. (2013). Estudio de los cambios de la actividad antioxidante en bebidas de café durante su periodo de vida útil usando métodos in-vitro y ex-vivo. Vitae, 20(2), 95-104.

Naranjo, M., Vélez, L., y Rojano, B. (2011). Actividad antioxidante de café colombiano de diferentes calidades. Revista Cubana Plantas Medicinales, 16(2), 164-173.

Tascón, O., y Sáenz, J. (2011). Ingeniería y café en Colombia. Revista de Ingeniería, 33(11), 99-114. 